\title{
e \\ Clinical and biochemical response to Denosumab in a young adult patient with craniofacial fibrous dysplasia
}

\author{
${ }^{1}$ E Cairoli, ${ }^{1} \mathrm{C}$ Eller-Vainicher C, ${ }^{2} \mathrm{DS}$ Rossi, ${ }^{2} \mathrm{GA}$ Beltramini, ${ }^{2} \mathrm{~A}$ Russillo, ${ }^{1} \mathrm{G}$ Mantovani, ${ }^{1} \mathrm{~A}$ Spada and ${ }^{1} \mathrm{I}$ Chiodini \\ ${ }^{1}$ Unit of Endocrinology and Metabolic Diseases, Fondazione IRCCS Ca' Granda - Ospedale Maggiore Policlinico, Department of Clinical \\ Sciences and Community Health, University of Milan, Milan, Italy. ${ }^{2}$ Department of Maxillofacial Surgery and Odontostomatology, \\ Fondazione IRCCS Ca' Granda - Ospedale Maggiore Policlinico, University of Milan, Milan, Italy.
}

\section{BACKGROUND}

Fibrous dysplasia (FD) is a rare condition in which normal bone and bone marrow are replaced by fibrous tissue and haphazardly distributed bone tissue, leading to fracture, functional impairment, deformity and pain. Nowadays, bisphosphonates therapy appears to be the only medical approach to manage FD-related bone pain, although the safety of a prolonged therapy in young adults is a matter of concern.

\section{CASE REPOR}

We present the case of a 20 -year-old male suffering from active craniofacial monostotic FD treated with the RANK-L inhibitor, Denosumab, following unsatisfactory responses to prior long-term bisphosphonates therapy. The patient had been treated over 9 years with pamidronic acid (cumulative dose of $81 \mathrm{mg}$ ), but he had experienced only mild reductions in pain scores.

Following initiation of Denosumab $60 \mathrm{mg}$ subcutaneously, bone pain and bone turnover markers (osteocalcin, total and bone alkaline phosphatase and $\beta$-crosslaps) were monitored over a period of 20 months. Following the first administration, the patient demonstrated a promptly and pronounced clinical and biochemical response: within few hours the patient reported pain disappearance and after 4 weeks bone turnover markers fell to levels within the normal range. Three months after initiation of Denosumab the patient reported an acute pain reactivation that required a second administration. As previously, after few hours the pain disappeared.

Later, Denosumab was administered according to pain reappearance and the injections were always followed by complete pain relief. However, a gradual shortening of the pain-free interval between administrations was observed, ranging from 90 days after the first one to 75 days after the sixth one.

Treatment with Denosumab has been always well tolerated.

The monitoring of bone turnover showed that all markers stayed in the lower half of the normal range, even at the moment of pain reappearance, suggesting that the effect of Denosumab on pain depends on mechanisms other than bone turnover suppression.
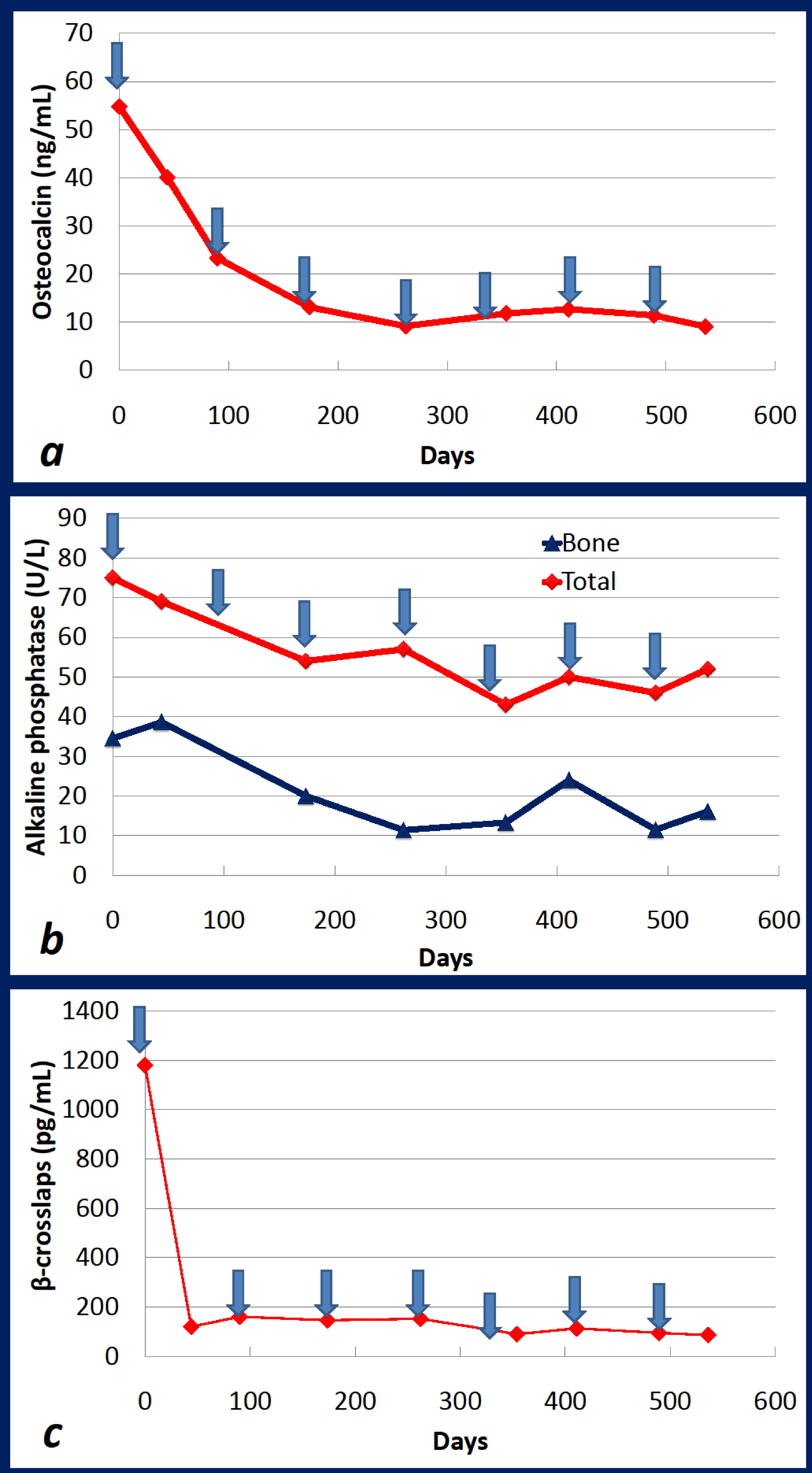

Changes in serum osteocalcin $(a)$, total and bone alkaline phosphatase $(b)$ and $\beta$-crosslaps $(c)$ in response to Denosumab treatment (reference range: osteocalcin $4.6-65.4 \mathrm{ng} / \mathrm{mL}$, total alkaline phosphatase $40-129 \mathrm{U} / \mathrm{L}, \quad \beta$-crosslaps $<584 \mathrm{pg} / \mathrm{mL}$ ).

Blue arrows indicate Denosumab administration.

CONCLUSIONS

Denosumab appears to be effective in reducing bone turnover and bone pain in adult patients with active monostotic FD and could represent a good alternative to bisphosphonates, allowing to avoid elevate cumulative doses of these agents.

Further studies are required to clarify the efficacy, safety and mechanisms of long-term therapeutic RANK-L inhibition in monostotic FD. 